\title{
Electron Holography of CMOS Devices with Epitaxial Layers
}

\author{
M. A. Gribelyuk, V. Ontalus, T. N. Adam, F. H. Baumann, P. R. Ronsheim \\ IBMSystems and Technology Group, Hopewell Junction, NY 12533, USA
}

Epitaxial SiGe and $\mathrm{SiC}(\mathrm{P})$ layers are used in the Source and Drain regions of the CMOS device to increase hole or electron mobility by imposing compressive (for p-FET device) or tensile (for n-FET device) stress in the channel region of the device [1,2]. Mapping of electrostatic potential in such devices with electron holography faces two new issues. First, the mean inner potential (MIP) of the epi layer Vepi(r) should be known, because it needs to be subtracted from the total potential available from the reconstructed phase image $\varphi(r)$ :

$$
V_{\text {dopant }}(r)=\varphi(r) /\left(C_{e} t\right)-V_{\text {epi }}(r)
$$

Here $\varphi(r)$ - phase image, $C_{\mathrm{e}^{-}}$interaction constant, $\mathrm{t}$ - sample thickness, $\mathrm{V}_{\mathrm{epi}}(\mathrm{r})-\mathrm{MIP}$ of the epi layer, $\mathrm{V}_{\text {dopant }}(\mathrm{r})$ - potential due to doping. Second, samples in holography analysis are tilted away from the zone axis by few degrees to minimize diffraction effects caused by Si or the epi layer. Therefore the epi layer overlaps with $\mathrm{Si}$ in the electron beam direction. Given that Si and the epi layer may have different MIP sample tilt makes it difficult to extract potential caused by doping from the phase image. After review of these problems the following results will be presented. All holography data was acquired on JEOL2010F TEM. The holography reconstruction package developed by Prof. Molly R. McCartney was used for reconstruction [3].

Measurement of MIP in $\mathrm{SiC}$ and $\mathrm{SiGe}$. MIP of $\mathrm{SiC}$ with the $\mathrm{C}=4 \%$ total Carbon content and $\mathrm{Cs}=1.4 \%$ Carbon substitutional fraction was measured from $\mathrm{SiC} / \mathrm{Si}$ structures in X-section. Both $\mathrm{SiC}$ and $\mathrm{Si}$ were undoped. Difference between MIP of $\mathrm{SiC}$ and $\mathrm{Si}$ was found below 0.1V, i.e. the measurement error of the experiment. MIP of undoped $\mathrm{Si}_{0.82} \mathrm{Ge}_{0.18}$ was measured using p-FET devices with epi layers for two cases of a strain state of SiGe, i.e. when it was either almost relaxed or its lattice was under compressive strain. The latter was achieved through formation of misfit dislocations at $\mathrm{Si} / \mathrm{SiGe}$ interface. Strain was measured from the same samples used for measurement of MIP. Strain was evaluated by Nano Beam Diffraction using F-strain algorithm [4]. Results indicate dependence of MIP of SiGe on the strain state (Table 1).

Analysis of Si:C films and Si:C based devices. Clusters formed by Si and interstitial Carbon have been observed in undoped $\mathrm{Si}_{1-\mathrm{x}} \mathrm{C}_{\mathrm{x}}$ films by conventional TEM. The cluster size increases with the total Carbon content, or concentration of the interstitial Carbon in the Si lattice. Electron holography has determined that clusters are negatively charged. Evidence of negative charging and an incomplete Phosphorus dopant activation was observed in n-FET devices, which were fabricated with the epitaxial $\mathrm{Si}_{1-\mathrm{x}} \mathrm{C}_{\mathrm{x}}(\mathrm{P})$ in the source and drain regions (Fig. 1)

Analysis of p-FET devices with epi-SiGe. Up to now sample tilt has restricted analysis of the dopant potential only to the regions close to the device channel, thus limiting its value. We suggest a method to correct for the sample tilt. It assumes that $\mathrm{Si} / \mathrm{SiGe}$ interface is compositionally sharp and the MIP of $\mathrm{SiGe}$ is known in advance. The width of the $\mathrm{Si} / \mathrm{SiGe}$ interface as viewed in the holography analysis and the position of the $\mathrm{Si} / \mathrm{SiGe}$ interface as viewed along the zone axis are derived from the reconstructed 
amplitude image. A parameterized erf-type function is fitted to the amplitude image to minimize effect of noise. These parameters are used then to correct potential image for tilt and the difference between MIP of Si and SiGe. As the result the dopant potential in the whole device can be evaluated (Fig. 2). Examples of analysis of dopant potential in p-FET devices and their correlation with electrical device data will be provided.

\section{References:}

[1] B. Jin, J. Brask, S. Datta, G. Dewey et.al. in Electrochem.Soc. Proc (2004) vol. 2004-07, pp. 111122

[2] B.(Frank) Yang, R. Takalkar, Z. Ren et.al., IEDM Tech.Dig., 51 (2008)

[3] M. R. McCartney, Software package for reconstruction in electron holography

[4] F. H. Baumann, F-STRAIN: Software algorithm for extraction of strain from nanobeam diffraction data (to be published)

[5] Authors acknowledge L. Kimball (IBM) for technical help.

\begin{tabular}{|l|l|l|l|}
\hline SiGe strain state & MIP, V & $\left(\mathrm{d}_{220} / \mathrm{d}_{220}{ }^{\mathrm{Si}}\right)-1, \%$ & $\left(\mathrm{~d}_{400} / \mathrm{d}_{400}{ }^{\mathrm{Si}}\right)-1, \%$ \\
\hline Almost relaxed & 12.85 & 0.6 & 0.7 \\
\hline $\begin{array}{l}\text { Under compressive } \\
\text { stress }\end{array}$ & 13.03 & -0.05 & 0.0 \\
\hline
\end{tabular}

Table 1. Mean Inner Potential of $\mathrm{Si}_{0.82} \mathrm{Ge}_{0 \cdot 18}$
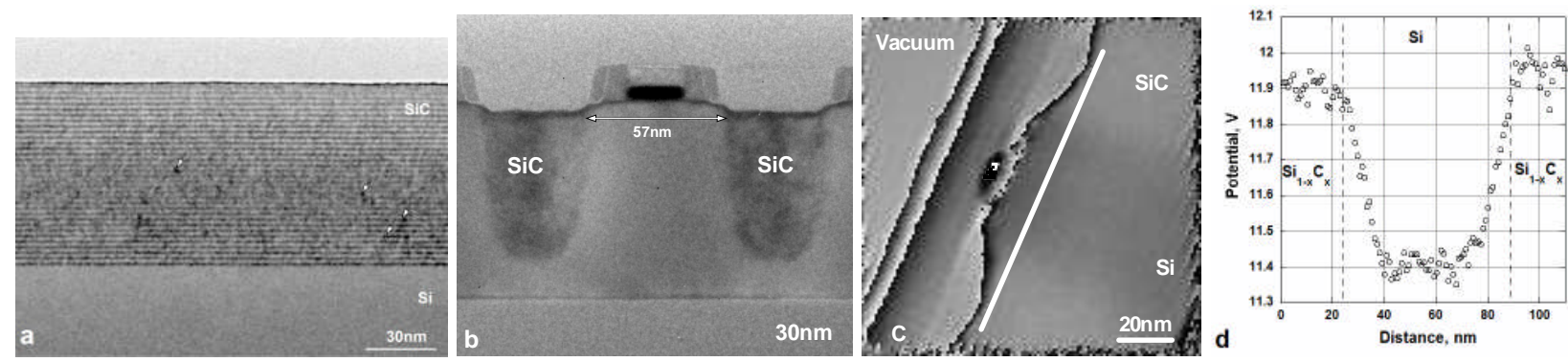

Fig. 1 (a): Conventional TEM image of $\mathrm{Si} / \mathrm{Si} 1-\mathrm{xCx}$ with substitutional Carbon fraction $\mathrm{Cs}=$ 1.4\%. Clusters in $\mathrm{Si}_{1-\mathrm{x}} \mathrm{C}_{\mathrm{x}}$ are arrowed; (b): n-FET device with similar Si:C film in Source-Drain regions; (c): Potential image of n-FET device; (d): Potential profile along the line shown in (c).
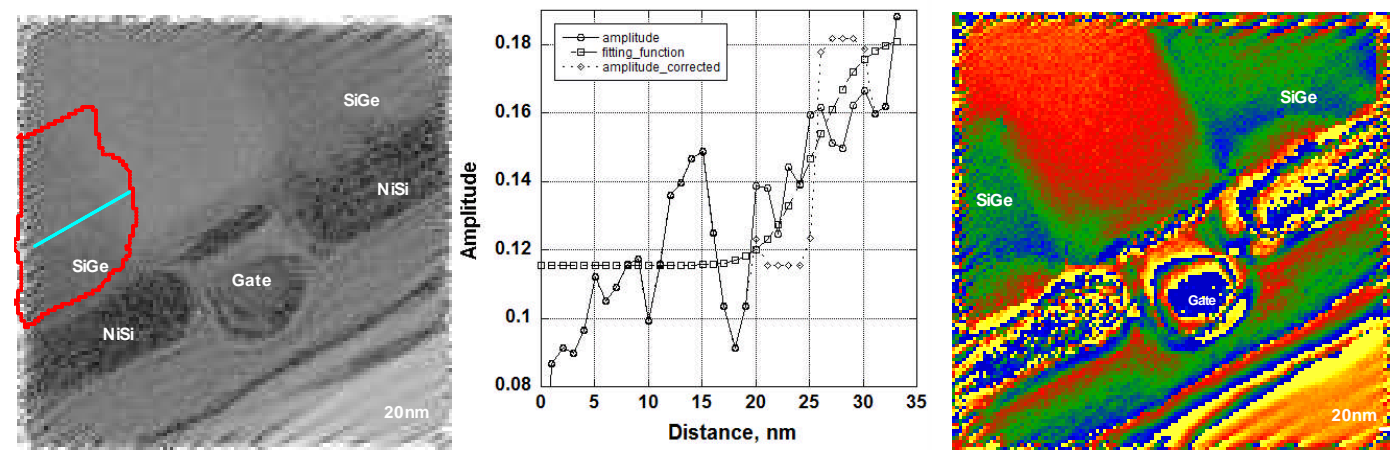

Fig.2. Left: amplitude map; Region for fitting is shown; Center: profile of amplitude, corrected amplitude and fitting function along the line shown in the amplitude image (left); Right: dopant potential after correction for sample tilt and difference of MIP between SiGe and Si. 